\title{
Harmonic Frequency Estimation Algorithm Based on MSWF in Power System
}

\author{
Cao Zhe", Sun Xiangwen and Niu Xinwen
}

School of Electrical Engineering, Henan University of Science and Technology, Luoyang, 471023, P.R. China

\begin{abstract}
This paper pointed out the disadvantage of harmonic frequency estimation algorithm in current power system, presented the MUSIC (multiple signal classification)-based harmonic frequency estimation algorithm in power system, and analyzed the computational complexity of the MUSIC algorithm. In order to reduce the computational complexity of conventional MUSIC algorithm and to increase the real-time characteristic of harmonic frequency estimation algorithm, we combined the multi-stage wiener filter (MSWF) recursive algorithm and MUSIC algorithm so as to avoid the subspace decomposition process of the conventional MUSIC algorithm, thus realizing the purpose of significantly reducing the computational complexity of the MUSIC algorithm. Through theoretical analysis and simulation experiments, we find that the algorithm proposed in this paper is of excellent resolution characteristic, and less dependent on data volume.
\end{abstract}

Keywords: Harmonic frequency estimation, MSWF, Rapid subspace decomposition.

\section{INTRODUCTION}

Over the past few years, with the rapid development of power electronic technology, more and more power electronic equipments have been introduced into power system, which causes an increasingly severe waveform distortion and the rising complexity of voltage and current in power system. When the harmonic component of power system surpasses the limitation standard, it will not only cause the effect decrease in power production, transmission and utilization, but also result in malfunctions of relay protection and automatic devices, leading to chaos of electric power measurement. In addition, harmonic frequency exerts severe interference to communication devices and electronic devices [1, 2], which will significantly affect the safety, reliability, stability and economical efficiency of the operation of power system and electric equipments. Conducting accurate and real-time detection on harmonic and interharmonic frequencies is the premise of solving harmonic problems. As a result, currently the method of rapidly estimating harmonic and interharmonic wave has become a hot research subject for worldwide researchers, and substantial research achievements have been made such as fast fourier transform (FFT), singular value decomposition (SVD) [3-5], wavelet transform (WT)[6], artificial neural network (ANN)[7] and multiple signal classification (MUSIC) algorithm [8].

In the case of analyzing multi-frequency signal spectrum using FFT algorithm, if the window width is the common multiple of signal cycles of various frequencies (i.e. in the synchronous sampling condition), we can obtain the accurate signal frequency spectrum. However, in practical situation, the power frequency normally fluctuates near industrial

\footnotetext{
*Address correspondence to this author at the School of Electrical Engineering, Henan University of Science and Technology, Luoyang, 471023, P.R. China; Tel: +86 13937941068; E-mail: cz7411@163.com
}

frequency and causes the non-synchronous sampling, under which, the spectrum leakage and picket fence effect will be easily caused and thus resulting big errors in harmonic estimation.

With regard to SVD, WT and neural network, they are superior in enhancing the accuracy of harmonic detection, but inferior for over high computational complexity that training is needed before operation and more prior information are aroused during operation, which cannot meet the requirements in meeting real-time harmonic detection.

MUSIC method is a kind of subspace algorithm, of which the basic principle is to decompose the signal characteristic space into signal subspace and the orthorhombic noise subspace using characteristic decomposition of autocorrelation matrix of sampled signals. Through taking the advantage of orthogonality between signal subspace and noise subspace, we built a pseudo spatial spectrum, and then estimated the unknown parameters based on the exploration of spectrum peak of pseudo spatial spectrum [9-11]. With the application of orthogonality of between signal subspace and noise subspace, this method is of high estimation accuracy and sound statistical performance by restraining the noise in largest degree, which is widely used in direction-of-arrival estimation, parameter estimation, and spatial spectrum estimation.

However, the computational complexity of this method is high and the causes mainly include: (1) MUSIC algorithm is a way of accurately estimating signal subspace and noise subspace. Normally, the estimation method of signal subspace and noise subspace is to compute the sample covariance matrix, and then conduct characteristic decomposition of it, wherein, the largest eigenvalue-based characteristic vectors expand into signal subspace, while the smallest eigenvalue-based characteristic vectors expand into noise subspace. As we all know that the computational 
complexity in characteristics decomposition of n-dimension matrix is $O\left(N^{3}\right)$, especially when $\mathrm{N}$ value is big, the computational amount will be astonishing. Based on national standard GB/T 14549-93 "harmonic power quality utility grid", the number of harmonic detection is prescribed as 19 as least, while based on requirement that prescribed in the new national standard GB/T 14549-2008 "harmonic power quality utility grid", the harmonic detection number should reach 50, and under this standard, the computational amount in direct characteristic decomposition of sample covariance matrix will be astonishing. (2)When using MUSIC algorithm to acquire the spatial spectrum peak, we need to conduct point by-point computation of spatial spectrum and then compare the computational results in the entire frequencydomain; Moreover if the requirement of estimation accuracy is high, the step distance during detection cannot be too large, otherwise the picket fence effect will occur, resulting in large computational errors.

Therefore in order to improve the real-time capability of MUSIC algorithm, we need to transform the MUSIC algorithm in terms of two aspects: (1) Seeking a fast and effective algorithm of subspace decomposition to replace the conventional eigenvalue decomposition algorithm. (2) Seeking a fast searching method of spectrum peaks to reduce the computational complexity in searching spectrum peaks.

This paper was written closely around above subject. Firstly, this paper introduced the MUSIC-based harmonic frequency estimation theory; Secondly, this paper introduced the method of obtaining signal subspace using MSWF-based subspace decomposition technique; Thirdly, this paper discussed the evaluation methods of $\mathrm{M}$ harmonic waves; Fourth, this paper introduced the strategy to accelerate searching of spatial spectral spectrum peaks using FFT; Lastly; this paper verified the proposed harmonic frequency estimation algorithm through numerical simulation experiment.

\section{SIGNAL MODEL}

Regarding the power system with $50 \mathrm{~Hz}$ industrial frequency, the frequency spectrum of voltage-current can be obtained by fourier decomposition, wherein the frequency spectrum with integral multiple of $50 \mathrm{~Hz}$ frequency is called harmonic wave. Therefore, in power system, the voltage and current sampling signal of noises, industrial frequencies and harmonic waves can be expressed as:

$$
x(n)=\sum_{i=1}^{M} A_{i} \cos \left(2 \pi f_{i} n+\phi_{i}\right)+e(n)
$$

wherein, $x(n)$ is the sampling signal, $A_{i}$ is the harmonic amplitude, $f_{i}$ is the normalization harmonic frequency, $n$ is the sampling time, $\phi_{i}$ is the initial phase of harmonic wave, $e(n)$ is the noise, $M$ is the number of harmonic wave.

For easiness to manage, order $\omega=2 \pi f$, and transform $x(n)$ into complex frequency signal, so equation (1) can be rewritten as:
$x(n)=\sum_{i=1}^{M} A_{i} e^{j\left(2 \omega_{i} n+\phi_{i}\right)}+e(n)$

Through adopting the sample data at point $\mathrm{N}$, we established Sampling data matrix $X$ at $K$ line and $(M<K) q$ column

$X=\left[\begin{array}{c}x_{1}(\mathrm{t}) \\ x_{2}(\mathrm{t}) \\ \vdots \\ x_{q}(\mathrm{t})\end{array}\right]^{T}=\left[\begin{array}{cccc}x(1) & x(2) & \vdots & x(q) \\ x(2) & x(3) & \vdots & x(q+1) \\ \ldots & \ldots & & \ldots \\ x(K) & x(K+1) & \vdots & x(N)\end{array}\right]$

wherein $x_{i}(\mathrm{t})=[x(i), x(i+1), \cdots x(i+K-1)]^{T} \in C^{K \times 1}$, is the number $i$ column of $X$,

Expressed as

$A=\left[\begin{array}{cccc}1 & 1 & \vdots & 1 \\ e^{j \omega_{1}} & e^{j \omega_{2}} & \vdots & e^{j \omega_{M}} \\ \cdots & \cdots & & \cdots \\ e^{j(K-1) \omega_{1}} & e^{j(K-1) \omega_{2}} & \vdots & e^{j(K-1) \omega_{M}}\end{array}\right] \in C^{K \times M}$

$S(\mathrm{t})=\left[\begin{array}{llll}A_{1} e^{j\left(n \omega_{1}+\varphi_{1}\right)} & A_{2} e^{j\left(n \omega_{2}+\varphi_{2}\right)} & \cdots & A_{M} e^{j\left(n \omega_{M}+\varphi_{M}\right)}\end{array}\right]^{T} \in C^{M \times 1}$

$E(\mathrm{t})=\left[\begin{array}{llll}e(n) & e(n+1) & \cdots & e(n+M-1)\end{array}\right]^{T} \in C^{M \times 1}$

According to the equation (2), we obtained that $x_{i}(\mathrm{t})=A S+E$, the autocorrelation matrix of $x_{i}(\mathrm{t})$

$R_{x}=\mathrm{APA}^{H}+\sigma^{2} I \in C^{K \times K}$

In the formula (3)

$\mathrm{P}=\operatorname{diag}\left\{\mathrm{A}_{1}^{2}, \mathrm{~A}_{2}^{2}, \cdots \mathrm{A}_{M}^{2}\right\} \in \mathrm{C}^{M \times M}$ 。

Based on matrix theory, $\operatorname{rank}\left(R_{x}\right)=M<K$, so $R_{x}$ should has $M$ nonzero eigenvalues, which can be sequenced from large to small as $\lambda_{1}, \lambda_{2} \cdots \lambda_{M}$, and the corresponding characteristic vectors $V_{1}, V_{2} \cdots V_{M}$ constituting the signal subspace $V_{s}=\left[V_{1}, V_{2}, \cdots, V_{M}\right]$. The remaining $K-M$ characteristic vector $\lambda_{i}$ 最 is the smallest and approximately equal, expressed as $\lambda_{M+1}=\lambda_{M+2}=\cdots=\lambda_{K}$, and the corresponding characteristic vectors $V_{M+1}, V_{M+2} \cdots V_{K}$ constituting the noise subspace $V_{n}=\left[V_{M+1}, V_{M+2}, \cdots, V_{K}\right]$, so

$R_{x}=V_{s} \sum_{s} V_{s}^{H}+\sigma_{n}^{2} V_{n} V_{n}^{H}$

\section{THE PRINCIPLE OF MUSIC-BASED HARMONIC FREQUENCY ESTIMATION ALGORITHM}

Constructing signal direction vector

$a(\omega)=\left[\begin{array}{llll}1 & e^{j \omega} & \cdots & e^{j(K-1) \omega}\end{array}\right]$

Since the signal component direction vector is orthogonally related with the noise subspace characteristic vector, the harmonic component can be expressed as 


$$
\begin{aligned}
& f(\omega)=a^{H}(\omega) V_{n} V_{n}^{H} a(\omega) \\
& \quad=a^{H}(\omega)\left(I-V_{s} V_{s}^{H}\right) a(\omega) \\
& \quad=0
\end{aligned}
$$

So, we can estimate the harmonic frequency through searching spatial spectrum peak.

$$
P_{\text {MUSIC }}=\frac{1}{f(\omega)} \quad \omega=1,2, \cdots \mathrm{M}
$$

\section{MSWF-BASED FAST SUBSPACE DECOMPOSITION}

As previously mentioned, in the case of estimating harmonic frequency by MUSIC algorithm, we need to conduct characteristic decomposition of sample autocorrelation matrix, in which the computational complexity is high and real-time processing is not easily to be realized. In order to reduce the computational complexity, many researchers have conducted lots of studies on this area, and achieved considerable valuable results including QR iteration, power method and Lanczos algorithm, by which the characteristic decomposition of sample autocorrelation matrix can be avoid and the computational complexity can be reduced in some degree, even though we still need to compute sample covariance matrix under the condition of suffering substantial computational complexities.

Proposed at 1997 by Goldstein et al., the MSWF is a kind of realization form of reduced-rank wiener filter, which consists of a decomposition filtering group and a composite filter group. Correspondingly, MSWF algorithm can be divided into forward recursive algorithm and backward recursive algorithm.

The basic steps of MSWF algorithm based on related subtraction structure are as follow:

1 , Constructing the data array by adopting the sampling data at $\mathrm{N}$ point

$$
X=\left[\begin{array}{c}
x_{1}(\mathrm{t}) \\
x_{2}(\mathrm{t}) \\
\vdots \\
x_{q}(\mathrm{t})
\end{array}\right]^{T}=\left[\begin{array}{cccc}
x(1) & x(2) & \vdots & x(q) \\
x(2) & x(3) & \vdots & x(q+1) \\
\ldots & \ldots & & \ldots \\
x(K) & x(K+1) & \vdots & x(N)
\end{array}\right]
$$

Define that the training signal as $d_{0}(k)=e_{1}^{T} x_{1}(k+\tau)$, the observed signal as $X_{0}=\left[\mathrm{x}_{2}, \mathrm{x}_{3}, \cdots, \mathrm{x}_{p}\right]^{T}$,

2, $\mathrm{M}$ time forward recursion:

$$
\begin{gathered}
\text { FOR } i=1,2, \cdots, M \\
h_{i}=E\left[d_{i-1}^{*}(k) X_{i-1}(\mathrm{k})\right] /\left\|E\left[d_{i-1}^{*}(k) X_{i-1}(\mathrm{k})\right]\right\|_{2} \\
d_{i}(k)=h_{i}{ }^{\mathrm{H}} X_{i-1}(k) \\
X_{i}(k)=X_{i-1}(k)-h_{i} d_{i}(k)
\end{gathered}
$$

So it can prove that, $\operatorname{span}\left\{h_{1}, h_{2}, \cdots h_{M}\right\}=V_{s}$
So, we can use MSWF to quickly compute the signal subspace $V_{s}$

What is needed to be emphasized is that the computational complexity of computing signal subspace based on MSWF forward recursive algorithm is O(MP2), approximately. While the computational complexity in computing sample covariance matrix and conducting eigenvalue decomposition is $\mathrm{O}(\mathrm{M} \mathrm{P} 2+\mathrm{P} 3)$. Therefore the MSWF forward recursive algorithm can significantly reduce the complexity in computing the signal subspace.

\section{ESTIMATION SIGNAL NUMBER}

As previously mentioned, before acquiring signal subspace by MSWF algorithm, the number of recursion should be determined (i.e the dimension of signal subspace), however, in practical situation, normally $\mathrm{M}$ cannot be directly obtained, which brings difficulty to the application of MSWF.

Theoretically, when the true covariance matrix $R_{x}$ is known, computing the characteristic value according to matrix theory, we can see that $P-M$ characteristic values $\lambda_{i}$ should be the smallest and equal, so that the accurate estimation value of $\mathrm{M}$ can be obtained.

However, in practical situation, the true covariance matrix can hardly be obtained, we can only get the approximately estimated $\widetilde{R_{x}}$, of which the smallest eigenvalues are non-equal to each other and distributed into the neighbourhood of noise variance. Therefore, we must estimate the number $\mathrm{M}$ of harmonic waves by more effective methods. Since the both common criterions (AIC criterion and MDL criterion) that are common used in the estimation of signal subspace dimension require the eigenvalue of $\widetilde{R_{x}}$, which means the computation and characteristic decomposition of $\mathrm{R}$ are necessarily to be done, resulting in the loss of significance in using MSWF algorithm to reduce the computational complexity.

According to the MSWF basic principle,

The output value $x_{i-1}(k)$ of the number $i$ MSWF can be processed as $d_{i}(k)=h_{i}^{H} x_{i-1}(k)$ by precondition matrix.

So, when the dimension of signal subspace is $\mathrm{m}$,

$$
d_{M}(k)=h_{M}{ }^{H} x_{M-1}(k) \quad, \quad d_{M+1}(k)=h_{M+1}{ }^{H} x_{M}(k)
$$

Obviously, in this occasion the correlation index between $d_{M}(k)$ and $d_{M+1}$ is 0 . On this basis, we can obtain the estimation value of M as well as the $\left\{h_{1}, h_{2}, \cdots h_{M}\right\}$ by MSWF.

\section{SPECTRUM PEAK SEARCHING}

After investigating the equation (6), we can see that $f(\omega)$ is the periodic function of $\omega$, and the Fourier expansion is 


$$
f(\omega)=\sum_{m=-\infty}^{\infty} F_{m} e^{j m \omega}
$$

In the formula (8) $F_{m}=\frac{1}{2 \pi} \int_{-\pi}^{+\pi} f(\omega) e^{-j m \omega} d \omega$

After cutting by $2 M-1$ points, we can get

$f(\omega) ; \sum_{m=-M}^{M} F_{m} e^{j m \omega}=\sum_{m=-M}^{M} F_{m} z^{m}=P(\mathrm{z})$

wherein, $z=e^{j \omega}$, through computing the root $e^{j m \omega}$ of equation $P(\mathrm{z})=0$ on the unit circle, we can estimate the harmonic angular frequency.

The coefficient $F_{m}$ of $P(\mathrm{z})$ can be approached by the fast Fourier transform (FFT) of $f(\omega)$

$\hat{F}_{m}=\frac{1}{2 \pi} \sum_{l=-(M-1)}^{M-1} f(l \Delta \omega) e^{-j m l \Delta \omega}$

In the equation, $\Delta \omega=\frac{2 \pi}{2 L-1}$ 。

Only when the $\mathrm{M}$ is big, the above algorithm can guarantee a truncation error during $f(\omega)$ 上 was truncated into $P(\mathrm{z})$, when $\mathrm{M}$ is relatively bigger, the computational complexity in the root process of equation $P(\mathrm{z})=0$ will increase.through the interpolation of null point, $P(\mathrm{z})$ can be converted into $2 J-1$ order, so

$$
\begin{aligned}
& P(\mathrm{z})=\sum_{m=-j+1}^{j-1} F_{m} e^{j m \omega}=\sum_{m=-(M-1)}^{M-1} F_{m} e^{j m \omega} \\
& =\sum_{m=-(M-1)}^{M-1} F_{m} z^{m}
\end{aligned}
$$

Through solving the root of $P(\mathrm{z})=0$ in equation on the unit circle, the harmonic frequency can be approximately evaluated.

In sum, the proposed harmonic frequency estimation algorithms are as follow:

1, Keep MSWF forward recursion till the correlation index between $d_{M}(k)$ and $d_{M+1}(k)$ equals 0 。

2, constructing the sampling signal subspace using the $\left\{h_{1}, h_{2}, \cdots h_{M}\right\}$ achieved by the first $\mathrm{M}$ recursions.

3, Constructing the spatial spectrum $P_{\text {MUSIC }}$ according to equation (6) and (7).

4, Based on the searching strategy proposed in section 6 , conduct spatial spectrum peak searching so as to obtain the estimation value of harmonic frequency.

\section{SIMULATION EXPERIMENT}

\section{Experiment 1}

Simulation experiment produce follow signals

$$
\begin{aligned}
x(t)= & 2 \cos (2 \pi \times 50 t+\pi / 4)+0.5 \cos (2 \pi \times 100 t+\pi / 5) \\
& +0.2 \cos (2 \pi \times 150 t+\pi / 6)
\end{aligned}
$$

Containing the signals of frequency components $50 \mathrm{~Hz}$, $100 \mathrm{~Hz}, 150 \mathrm{~Hz}$, without noise.

In the experiment, the sampling frequency $f_{s}=1000 \mathrm{~Hz}$, experimental period is $50 \mathrm{~ms}$, sampling numbers $\mathrm{N}=50$, sampling sequence was estimated by FFT algorithm and the proposed algorithm, respectively, and the results were shown in Fig. (1)

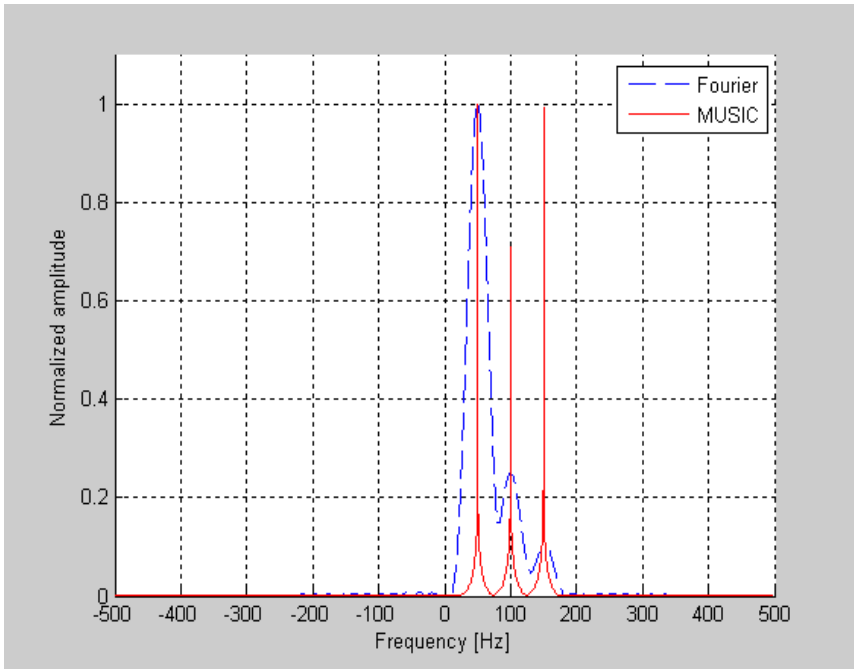

Fig. (1). Time series and spectrum analysis results.

According to the experiment results we can see the under the synchronous sampling and noise free condition, the performance of the proposed algorithm is similar to that of FFT algorithm, both can accurately estimate the harmonic component of signals under test.

\section{Experiment 2}

Simulation experiment produce follow signals

$$
\begin{aligned}
x(t)= & 2 \cos (2 \pi \times 35 t+\pi / 4)+0.5 \cos (2 \pi \times 50 t+\pi / 5) \\
& +0.2 \cos (2 \pi \times 70 t+\pi / 6)
\end{aligned}
$$

Containing the signals of frequency components $35 \mathrm{~Hz}$, $50 \mathrm{~Hz}, 70 \mathrm{~Hz}$, without noise.

In the experiment, the sampling frequency $f_{s}=1000 \mathrm{~Hz}$, experimental period is $15 \mathrm{~ms}$, sampling numbers $\mathrm{N}=16$, sampling sequence was estimated by FFT algorithm and the 
proposed algorithm, respectively, and the results were shown in Fig. (2).

As we all know that, the frequency resolution of FFT algorithm mainly depends on the sampling data and the data volume, in this experiment $f_{s} / N=62.5 \mathrm{~Hz}$, due to the small amount of sampling numbers, the identifying and detection processes for $45 \mathrm{~Hz}$ and $70 \mathrm{~Hz}$ signals can hardly be achieved.

Under the same condition, the proposed algorithm can accurately detect and estimate the frequency of each harmonic signal.

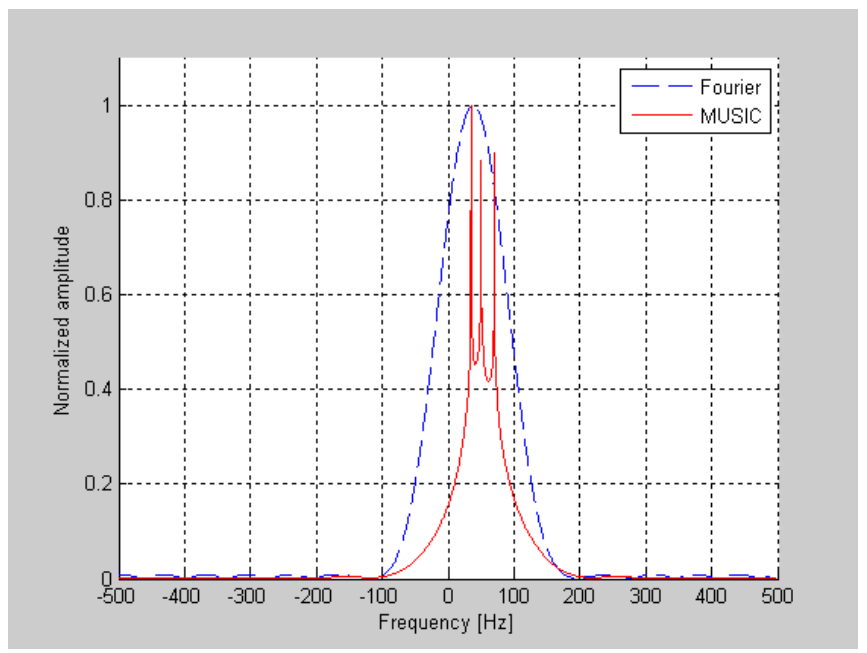

Fig. (2). Time series and spectrum analysis results.

\section{Experiment 3}

Through adding gaussian white noise in the condition of experiment 1 , the condition of experiment 3 was achieved ( $\mathrm{SNR}=30$, sampling frequency remained unchanged), under which the simulation signals were produced, and we tested the performance of the proposed algorithm under the low SNR condition, and the results were shown in Fig. (3).

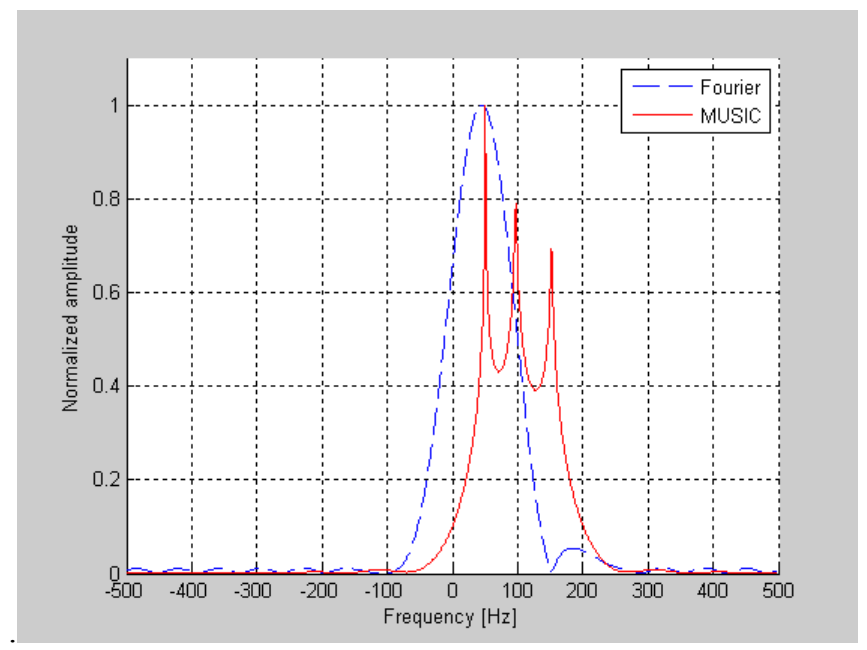

Fig. (3). Time series and spectrum analysis results.
Comparing the Figs. $(\mathbf{1}, \mathbf{3})$, we can see that the FFT algorithm is very sensitive to SNR, while the proposed algorithm remained roughly unchanged in the condition that SNR sharply decreased. This experiment indicates the proposed algorithm is of strong noise resistant capacity, which is very important in practical application.

\section{CONCLUSION}

MUSIC algorithm is one of most effective method for frequency estimation, delay estimation, and direction-ofarrival estimation. However, during operation of this algorithm, it is needed to conduct characteristic decomposition of autocorrelation matrix of sampling signals as well as to search the spatial spectrum peak; therefore the computational complexity is high. Integrated with MSWFbased fast subspace decomposition technique, FFT-based fast spectrum peak searching technique and MUSIC, the algorithm proposed in this paper can significantly reduce the computational complexity without affecting the estimation accuracy. The simulation results show that the proposed algorithm is of sound noise resistant effect and can realize the high resolution harmonic frequency estimation. Even without synchronous sampling, the proposed algorithm can perfectly meet the requirement of harmonic frequency estimation in power system without causing the problem of spectrum leakage, which possesses unmatched superiority over the classic Fourier analysis method.

\section{CONFLICT OF INTEREST}

The authors confirm that this article content has no conflict of interest.

\section{ACKNOWLEDGEMENTS}

Declared none.

\section{REFERENCES}

[1] T. Tang, Z. Wu, and S. Sun, "Harmonious Waves in Power Systems," China Mining University Press, 1991.

[2] K. Xu, Y. Xu, and F. Liu, "Ultraharmonics in Power System," Chongqing University Press, 1991.

[3] Q. Jin, and A. Peng, "FFT-based Detecting Algorithm of Noninteger Harmonic Parameters," Microcomputer Information, no.22, pp. 212-213, 2006

[4] T. Lobos, T. Kozina, and H Koglin, "Power system harmonics estimation using linear least squares method and SVD," In: IEE Proceedings Generation, Transmission and Distribution, vol. 148, no.6, pp. 567-572, 2001.

[5] L. Zhou, X. Xia, and Y. Wan, "Overview of Harmonic Measurement Methods Based on Wavelet Transform," Trans. China Electrotech. Soc., vol. 21, no.9, pp. 67-74, 2006.

[6] J.S. Goldstein, I. S. Reed, and L.L. Scharf, "A multistage representation of the wiener filter Based on orthogonal projections," IEEE Trans Inform. Theory, vol. 44, no.7, pp. 2943 2959, 2002.

[7] R.O. Schmidt, "Multiple emitter location and signal parameter estimation," IEEE Trans. Antennas Propag., vol. 34, no.3, pp. 276$280,1986$. 
[8] L. Huang, S. Wu, L. Zhang, and D. Feng, "Fast Subspace Decompostion Method and Fast Estimation Method of Subspace Dimension," Chin. J. Electron., vol. 33, no.6, pp. 977-981, 2005.

[9] X. Wang, and N. Li, "Research of Interharmonic Detection Methods Based on Windowed Interpolation FFT Algorithm," Mod. Electric. Power, vol. 29, no.5, pp. 28-31, 2012.
[10] Q. Ding, H. Cheng, and G. Lv, "Harmonic and Interharmonic Frequency Spectrum Estimation Based on Prony Algorithm," Trans. China Electrotech. Soc., vol. 20, no.10, pp. 94-97, 2005.

[11] M. L. Honig, and W. Xiao, "Performance of reduced 2 rank linear interference suppression," IEEE Trans Inform. Theory, vol. 47, no.5, pp.1928-1946, 2001.

(C) Zhe et al.; Licensee Bentham Open.

This is an open access article licensed under the terms of the Creative Commons Attribution Non-Commercial License (http://creativecommons.org/licenses/by$\mathrm{nc} / 3.0 /$ ) which permits unrestricted, non-commercial use, distribution and reproduction in any medium, provided the work is properly cited. 